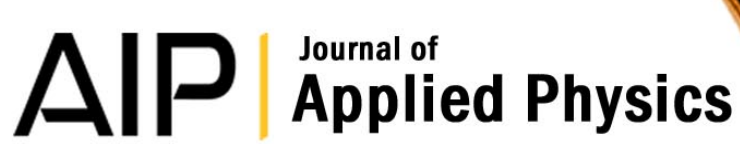

\section{Primary and secondary pyroelectric effects of ferroelectric 0-3 composites}

K.-H. Chew, F. G. Shin, B. Ploss, H. L. W. Chan, and C. L. Choy

Citation: J. Appl. Phys. 94, 1134 (2003); doi: 10.1063/1.1583154

View online: http://dx.doi.org/10.1063/1.1583154

View Table of Contents: http://jap.aip.org/resource/1/JAPIAU/v94/i2

Published by the AIP Publishing LLC.

\section{Additional information on J. Appl. Phys.}

Journal Homepage: http://jap.aip.org/

Journal Information: http://jap.aip.org/about/about_the_journal

Top downloads: http://jap.aip.org/features/most_downloaded

Information for Authors: http://jap.aip.org/authors

\section{ADVERTISEMENT}

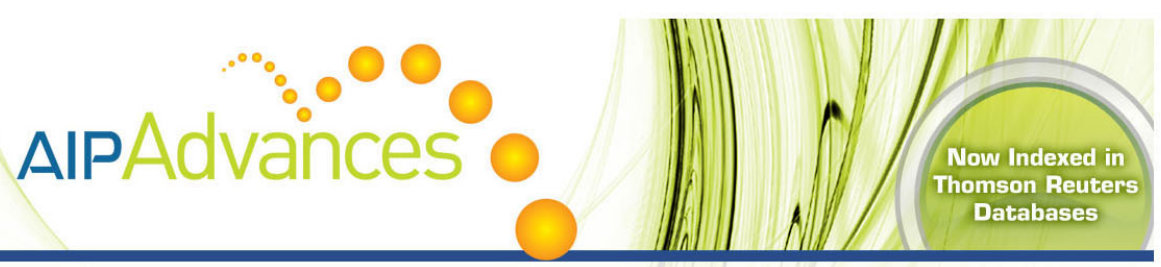

\section{Explore AIP's open access journal: Rapid publication \\ - Article-level metrics \\ - Post-publication rating and commenting}




\title{
Primary and secondary pyroelectric effects of ferroelectric 0-3 composites
}

\author{
K.-H. Chew, ${ }^{\text {a) }}$ F. G. Shin, B. Ploss, H. L. W. Chan, and C. L. Choy \\ Department of Applied Physics, The Hong Kong Polytechnic University, Hung Hom, Kowloon, Hong Kong
}

(Received 11 December 2002; accepted 28 April 2003)

\begin{abstract}
Simple and tractable analytical expressions for determining the pyroelectricity in ferroelectric $0-3$ composites have been developed. For the dilute suspension limit, expressions for the effective pyroelectric and other thermal electromechanical properties are derived within the framework of the Maxwell-Wagner approach. Then, an effective-medium theory is employed to examine the first and second pyroelectric coefficients in the concentrated suspension regime. The effective-medium approach as compared to the Maxwell-Wagner approach results in a better agreement with known experimental data up to higher volume fraction of inclusions. The pyroelectricity of 0-3 composites of ceramic inclusions embedded in the $\mathrm{P}(\mathrm{VDF}-\mathrm{TrFE})$ copolymer matrix and of $\mathrm{P}(\mathrm{VDF}-\mathrm{TrFE})$ inclusions embedded in a ceramic matrix are analyzed numerically under different polarization configurations. The theoretical predictions show that the secondary pyroelectric effects in composite systems with ceramic as the matrix are stronger than those with the copolymer as the matrix and can sometimes dominate the pyroelectricity for certain compositions. (C) 2003 American Institute of Physics. [DOI: 10.1063/1.1583154]
\end{abstract}

\section{INTRODUCTION}

Pyroelectric materials are extensively used for thermal infrared detectors ${ }^{1}$ due to advantages such as good sensitivity, room temperature operation, and low cost. $^{2}$ In recent years, the research in the field of pyroelectricity has been concentrated on discovering materials with higher figures of merit. One way to attain this is to dilute the ferroelectric material in order to reduce the dielectric constant as observed in sol-gel processed $(\mathrm{Pb}, \mathrm{Ca}) \mathrm{TiO}_{3} \cdot{ }^{3}$ Composite materials of ferroelectric ceramics particles embedded in polymer matrices with different connectivities ${ }^{4}$ aroused great interest because of their mechanical flexibility, low cost, and excellent pyroelectricity. ${ }^{5}$ Another advantage is that their dielectric, thermal, and pyroelectric properties can be changed by varying the volume fraction of ceramic particles or poling procedure. $^{6}$

Many experimental works have been done on the pyroelectric properties of ferroelectric composites. ${ }^{7}$ Theoretically, Bhalla et al. investigated the primary and secondary pyroelectric effects and derived expressions for the effective pyroelectric coefficients based on simple series and parallel connections. ${ }^{5}$ Yamazaki et al. derived the pyroelectric coefficient of 0-3 composites with identical spherical ferroelectric particles. ${ }^{8}$ Based on a modified Clausius-Mossotti relation, ${ }^{9}$ Wang et al. ${ }^{10}$ formulated the pyroelectric coefficient of ferroelectric composites by taking into account the depolarization coefficient. $\mathrm{Nan}^{11}$ developed a formulation to describe the pyroelectricity of ferroelectric composite materials. The theoretical framework is developed in terms of a Green's function method and perturbation theory which is more rigorous but complicated. Recently, Levin and Luchaninov have derived expressions for the effective pyroelectric constants of composite materials with spherical

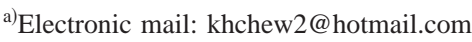

inclusion ${ }^{12}$ by means of effective field theory. While extensive theoretical studies ${ }^{8-12}$ have been made on the pyroelectricity of 0-3 composites, the works are mainly concerned with the effective pyroelectric effect of ferroelectric composites and not explicitly on the secondary effect.

This work concerns deriving explicit analytical expressions for the primary and secondary pyroelectric effects of ferroelectric 0-3 composites. We employ two methods in succession in this study: the Maxwell-Wagner (MW) approach and then effective-medium (EM) theory; the former gives expressions for the case of a dilute suspension of inclusion particles while the latter gives results for a wider range of particle concentration. Section II contains the general expressions for the primary and secondary effects of ferroelectric 0-3 composites. The definition of pyroelectricity is given, followed by a detailed derivation of primary and secondary pyroelectric coefficients within the framework of the two methods mentioned earlier. In Sec. III, the pyroelectricity of composite systems consisting of ferroelectric ceramic and polymer phases is examined. Comparison with experimental results is performed to verify the two theoretical models. Some conclusions are given in Sec. IV.

\section{THEORY}

Pyroelectricity is the electrical response of a polar material as a result of a change in temperature. The pyroelectric effects at constant stress $\boldsymbol{\sigma}$ and electric field $\mathbf{E}$ are defined as ${ }^{13}$ confining to scalar notations,

$$
\left(\frac{\partial \mathbf{D}}{\partial T}\right)_{E, \sigma}=\left(\frac{\partial \mathbf{D}}{\partial T}\right)_{E, e}+\left(\frac{\partial \mathbf{D}}{\partial \mathbf{e}}\right)_{E, T}\left(\frac{\partial \mathbf{e}}{\partial T}\right)_{E, \sigma},
$$

where $\mathbf{D}$, e, and $T$ represent, respectively, the electric displacement, strain, and temperature of the material. The term $(\partial \mathbf{D} / \partial T)_{E, e}$ of Eq. (1) is called the primary pyroelectric response. The primary pyroelectric effect corresponds to the 
charges produced owing to the change in polarization with temperature when the dimensions of the pyroelectric material are fixed. For a material under constant stress, its dimensions change with temperature due to thermal expansion resulting in an additional contribution of piezoelectrically induced charge. This leads to the secondary pyroelectric effect which is defined as the final term of Eq. (1).

For ferroelectric materials, the electric displacement can be related as

$$
\mathbf{D}=\mathbf{P}+\mathbf{d} \boldsymbol{\sigma},
$$

with the polarization term $\mathbf{P}$ contributed by the "switchable" polarization $\mathbf{P}_{S}$ and electric field $\mathbf{E}$ given by

$$
\mathbf{P}=\mathbf{P}_{S}+\varepsilon \mathbf{E} \text {. }
$$

In Eqs. (2) and (3), $\mathbf{d}$ is the piezoelectric coefficient and $\varepsilon$ the permittivity of the material.

From Eqs. (1) to (3), the primary pyroelectric coefficient is expressed as

$$
\rho_{1} \equiv\left(\frac{\partial \mathbf{D}}{\partial T}\right)_{E, \sigma}=\left(\frac{\partial \mathbf{P}}{\partial T}\right)_{E, \sigma}=\frac{\partial \mathbf{P}_{S}}{\partial T}+\mathbf{E} \frac{\partial \varepsilon}{\partial T},
$$

where the first term $\partial \mathbf{P}_{S} / \partial T$ represent $\rho_{1}$ at zero applied electric field at a temperature below the transition temperature $T_{C}$. The second term exploits the $\rho_{1}$ near the Curie temperature where $\partial \varepsilon / \partial T$ can be quite large. The secondary pyroelectric coefficient is written as

$\rho_{2} \equiv\left(\frac{\partial \mathbf{D}}{\partial \mathbf{e}}\right)_{\mathbf{E}, T}\left(\frac{\partial \mathbf{e}}{\partial T}\right)_{\mathbf{E}, \sigma}=\left(\frac{\partial \mathbf{D}}{\partial \boldsymbol{\sigma}}\right)_{\mathbf{E}, T}\left(\frac{\partial \boldsymbol{\sigma}}{\partial \mathbf{e}}\right)_{\mathbf{E}, T}\left(\frac{\partial \mathbf{e}}{\partial T}\right)_{\mathbf{E}, \sigma}=\mathbf{d c} \boldsymbol{\alpha}$,

which is the contribution induced by the coupled effects of thermal deformation and piezoelectricity. $c$ and $\alpha$ are the elastic stiffness and thermal expansion coefficients of the pyroelectric material. In the present study, explicit expressions for both the primary and secondary pyroelectric responses of ferroelectric 0-3 composites are derived with examples given for $T$ sufficiently far below $T_{C}$ of the two constituents, where the pyroelectric contribution from the dielectric permittivity is weak and can be neglected.

\section{A. Single spherical inclusion in a homogeneous pyroelectric matrix}

Consider the single inclusion problem of a spherical inclusion embedded in an infinite matrix, in which both materials are ferroelectric as well as dielectrically and elastically isotropic and homogeneous, as shown in Fig. 1. We assume that both the constituents may be under hydrostatic stresses as may be created by thermal expansion. Under the action of a weak electric field $E_{m}$ in the matrix region far away from the inclusion, the electric displacements and electric fields in the two materials are related by the following expression (Appendix A):

$$
\left\langle D_{i}\right\rangle+2 \varepsilon_{m}\left(\left\langle E_{i}\right\rangle-\left\langle E_{m}\right\rangle\right)=\left\langle D_{m}\right\rangle,
$$

with

$$
\left\langle D_{i}\right\rangle=\left\langle P_{i}\right\rangle+\varepsilon_{i}\left\langle E_{i}\right\rangle+d_{h i}\left\langle\sigma_{i}\right\rangle,
$$

and

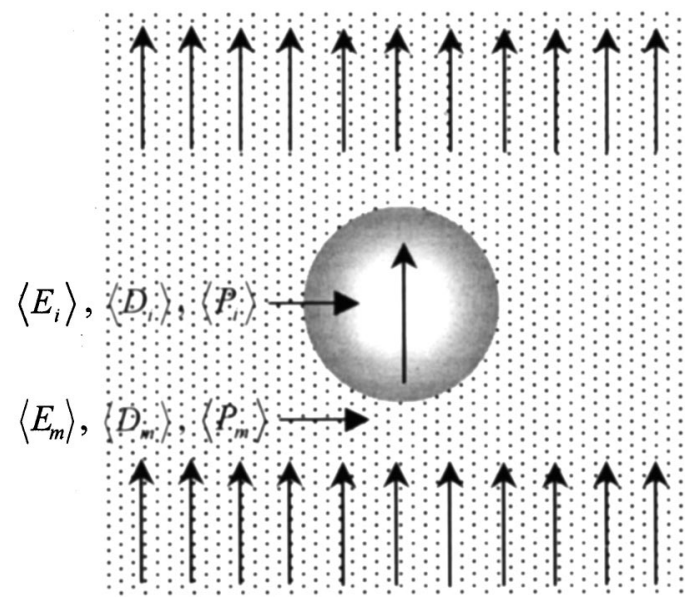

FIG. 1. Spherical inclusion embedded in matrix phase of permittivity $\varepsilon_{m}$. The volume average dielectric permittivity, polarization and electric field are represent as $\langle D\rangle,\langle P\rangle$, and $\langle E\rangle$, respectively. The spherical inclusion and matrix are denoted by subscripts $i$ and $m$, respectively.

$$
\left\langle D_{m}\right\rangle=\left\langle P_{m}\right\rangle+\varepsilon_{m}\left\langle E_{m}\right\rangle+d_{h m}\left\langle\sigma_{m}\right\rangle,
$$

where $\langle\zeta\rangle=1 / V \int \zeta d V$ denotes the volumetric averaging of the quantity $\zeta$. Subscripts $i$ and $m$ refer to inclusion and matrix. $P, \varepsilon, d_{h}$, and $\sigma$ represent the polarization, dielectric permittivity, piezoelectric coefficient, and hydrostatic stress, respectively.

The hydrostatic stresses are related by static equilibrium conditions in a similar way as can be obtained by manipulating with standard results such as given by Christensen ${ }^{14}$ (Appendix B), thus

$$
\left\langle\sigma_{m}\right\rangle=\left\langle\sigma_{i}\right\rangle+\frac{4}{3} \mu_{m}\left(\left\langle e_{i}\right\rangle-\left\langle e_{m}\right\rangle\right),
$$

where $e$ is volumetric strain and $\mu$ is shear modulus.

For deformation involving a change of temperature $\Delta T$, the relationship between the hydrostatic stress, volumetric strain, and temperature of the inclusion is ${ }^{15}$

$$
\left\langle\sigma_{i}\right\rangle=k_{i}\left(\left\langle e_{i}\right\rangle-\alpha_{i} \Delta T\right),
$$

where $k$ and $\alpha$ represent the bulk modulus and the thermal expansion coefficient of the material, respectively. Here, $\Delta T \equiv T-T_{\text {ref }}$ is the temperature difference from a reference temperature $T_{\text {ref }}$ at which thermal stress does not arise or taken as zero. A similar equation may be written for the matrix material.

\section{B. Maxwell-Wagner (MW) approach for deriving the effective constants of pyroelectric 0-3 composites in the dilute suspension limit}

Consider now a composite material consisting of a welldispersed, dilute suspension of spherical pyroelectric inclusions in a pyroactive matrix phase, the properties of which are to be determined. Imagine a single sphere of such a composite material is embedded in an infinite matrix of the original matrix phase (Fig. 2). This constitutes again the single inclusion problem discussed above. This is so because the sphere of the composite material may be considered to be a 


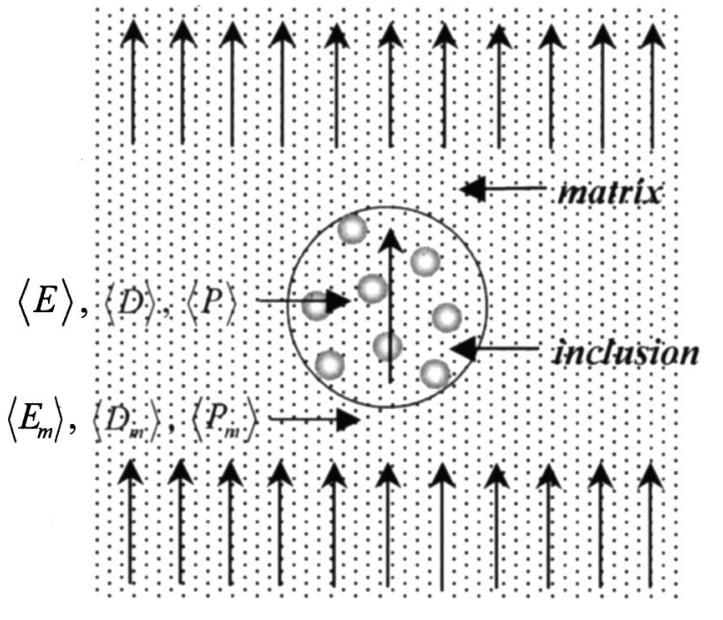

FIG. 2. Maxwell-Wagner model consists of a spherical inclusion of the composites material surrounded by matrix phase. The volume-averaged dielectric permittivity, polarization, and electric field are represent as $\langle D\rangle$, $\langle P\rangle$, and $\langle E\rangle$, respectively. The spherical inclusion and matrix are denoted by subscripts $i$ and $m$, respectively.

homogenous sphere of an equivalent material having the effective properties of the composite. Thus, for the present problem, the analog of Eq. (6) is

$$
\langle D\rangle+2 \varepsilon_{m}\left(\langle E\rangle-\left\langle E_{m}\right\rangle\right)=\left\langle D_{m}\right\rangle,
$$

where quantities without subscripts relate to the composite. Similarly, a result analogous to Eq. (9) on hydrostatic stress is

$$
\langle\sigma\rangle+\frac{4}{3} \mu_{m}\left(\langle e\rangle-\left\langle e_{m}\right\rangle\right)=\left\langle\sigma_{m}\right\rangle .
$$

The dielectric permittivity $\varepsilon$ and hydrostatic piezoelectric coefficient $d_{h}$ of the composite are related to the electric displacement $\langle D\rangle$, polarization $\langle P\rangle$, applied field $\langle E\rangle$, and stress $\langle\sigma\rangle$ as

$$
\langle D\rangle=\langle P\rangle+\varepsilon\langle E\rangle+d_{h}\langle\sigma\rangle,
$$

and the deformation to a change in temperature $\Delta T$ and hydrostatic stress as ${ }^{15}$

$$
\langle\sigma\rangle=k(\langle e\rangle-\alpha \Delta T),
$$

where $k$ and $\alpha$ represent the bulk modulus and thermal expansivity of the composite material, respectively.

From Eqs. (9) and (12), we obtain another form of stress-strain relationship as

$$
\langle\sigma\rangle+\frac{4}{3} \mu_{m}\langle e\rangle=\left\langle\sigma_{i}\right\rangle+\frac{4}{3} \mu_{m}\left\langle e_{i}\right\rangle .
$$

The electric field in the inclusion can be obtained by solving Eqs. (6), (7), and (11), thus

$$
\left\langle E_{i}\right\rangle=\frac{\langle D\rangle+2 \varepsilon_{m}\langle E\rangle-\left\langle P_{i}\right\rangle-d_{h i}\left\langle\sigma_{i}\right\rangle}{\varepsilon_{i}+2 \varepsilon_{m}} .
$$

Manipulations of Eqs. (10), (14), and (15) provide the expression for stress acting on the inclusion as

$$
\left\langle\sigma_{i}\right\rangle=\langle\sigma\rangle\left(\frac{1+\frac{4 \mu_{m}}{3 k}}{1+\frac{4 \mu_{m}}{3 k_{i}}}\right)+\left(\alpha-\alpha_{i}\right) \Delta T\left(\frac{\frac{4 \mu_{m}}{3}}{1+\frac{4 \mu_{m}}{3 k_{i}}}\right),
$$

as well as the relationship between the strain of the inclusion $\left\langle e_{i}\right\rangle$ and temperature change $\Delta T$ as

$$
\left\langle e_{i}\right\rangle=\frac{\left(k+\frac{4}{3} \mu_{m}\right)\langle e\rangle-\left(k \alpha-k_{i} \alpha_{i}\right) \Delta T}{\left(k_{i}+\frac{4}{3} \mu_{m}\right)} .
$$

We are now ready to solve the pyroelectric coefficients of the pyroelectric composite material. From Eq. (C6) (Appendix C) together with Eqs. (10) and (14), we have

$$
\begin{aligned}
& \left(k-k_{m}\right)\langle e\rangle-\left(k \alpha-k_{m} \alpha_{m}\right) \Delta T \\
& \quad=\phi\left[\left(k_{i}-k_{m}\right)\left\langle e_{i}\right\rangle-\left(k_{i} \alpha_{i}-k_{m} \alpha_{m}\right) \Delta T\right] .
\end{aligned}
$$

Substitution of $\left\langle e_{i}\right\rangle$ into Eq. (19) and by equating terms involving $\langle e\rangle$ and $\Delta T$ give

$$
\left(k-k_{m}\right)=\phi\left(k_{i}-k_{m}\right)\left(\frac{k+\frac{4}{3} \mu_{m}}{k_{i}+\frac{4}{3} \mu_{m}}\right),
$$

and

$$
\begin{aligned}
\left(k \alpha-k_{m} \alpha_{m}\right)= & \phi\left(k_{i}-k_{m}\right)\left(\frac{k \alpha-k_{i} \alpha_{i}}{k_{i}+\frac{4}{3} \mu_{m}}\right) \\
& +\phi\left(k_{i} \alpha_{i}-k_{m} \alpha_{m}\right) .
\end{aligned}
$$

After some mathematical manipulation, Eq. (20) gives the bulk modulus of the composite which is similar to Hashin's ${ }^{16}$ as

$$
\frac{1}{k}=\frac{\frac{1}{k_{i}}\left(\frac{\phi}{1+4 \mu_{m} / 3 k_{i}}\right)+\frac{1}{k_{m}}\left(\frac{1-\phi}{1+4 \mu_{m} / 3 k_{m}}\right)}{\left(\frac{\phi}{1+4 \mu_{m} / 3 k_{i}}\right)+\left(\frac{1-\phi}{1+4 \mu_{m} / 3 k_{m}}\right)},
$$

and Eq. (21) together with Eq. (22) give Levin's ${ }^{17}$ expansion coefficient formula in the form

$$
\alpha-\alpha_{i}=\left(\alpha_{m}-\alpha_{i}\right)\left(\frac{1 / k_{i}-1 / k}{1 / k_{i}-1 / k_{m}}\right) .
$$

Using Eq. (C3) together with Eqs. (7), (16), and (17) gives

$$
\begin{aligned}
(1- & \left.\phi \frac{\varepsilon_{i}-\varepsilon_{m}}{\varepsilon_{i}+2 \varepsilon_{m}}\right)\langle D\rangle \\
= & \phi\left(\frac{3 \varepsilon_{m}}{\varepsilon_{i}+2 \varepsilon_{m}}\right)\left\langle P_{i}\right\rangle+(1-\phi)\left\langle P_{m}\right\rangle \\
& +\phi\left(\left(\frac{3 \varepsilon_{m}}{\varepsilon_{i}+2 \varepsilon_{m}}\right) d_{h i}-d_{h m}\right)
\end{aligned}
$$




$$
\begin{aligned}
& \times\left(\alpha-\alpha_{i}\right) \Delta T\left(\frac{\frac{4 \mu_{m}}{3}}{1+\frac{4 \mu_{m}}{3 k_{i}}}\right) \\
& +\left(2 \varepsilon_{m} \phi\left(\frac{\varepsilon_{i}-\varepsilon_{m}}{\varepsilon_{i}+2 \varepsilon_{m}}\right)+\varepsilon_{m}\right)\langle E\rangle \\
& +\left\{\begin{array}{l}
\left.d_{h m}+\phi\left(\frac{3 \varepsilon_{m}}{\varepsilon_{i}+2 \varepsilon_{m}}\right) d_{h i}-d_{h m}\right) \\
1+\frac{4 \mu_{m}}{3 k} \\
\frac{4 \mu_{m}}{3 k_{i}}
\end{array}\right\}\langle\sigma\rangle .
\end{aligned}
$$

Comparing Eq. (24) with Eq. (13), we obtain the total polarization $\langle P\rangle$, effective permittivity $\varepsilon$, and effective piezoelectric coefficient $d_{h}$ of the composite within the MW (Ref. 18) framework as

$$
\begin{aligned}
& \langle P\rangle=\phi\left(\frac{\frac{3 \varepsilon_{m}}{\varepsilon_{i}+2 \varepsilon_{m}}}{1-\phi \frac{\varepsilon_{i}-\varepsilon_{m}}{\varepsilon_{i}+2 \varepsilon_{m}}}\right)\left\langle P_{i}\right\rangle \\
& +(1-\phi)\left(\frac{1}{1-\phi \frac{\varepsilon_{i}-\varepsilon_{m}}{\varepsilon_{i}+2 \varepsilon_{m}}}\right)\left\langle P_{m}\right\rangle \\
& \phi \frac{\left(\frac{3 \varepsilon_{m}}{\varepsilon_{i}+2 \varepsilon_{m}} d_{h i}-d_{h m}\right)\left(\alpha-\alpha_{i}\right)\left(\frac{\frac{4 \mu_{m}}{3}}{1+\frac{4 \mu_{m}}{3 k_{i}}}\right)}{1-\phi \frac{\varepsilon_{i}-\varepsilon_{m}}{\varepsilon_{i}+2 \varepsilon_{m}}} \Delta T,
\end{aligned}
$$

$$
\varepsilon=\left(\frac{\varepsilon_{m}+\phi 2 \varepsilon_{m} \frac{\varepsilon_{i}-\varepsilon_{m}}{\varepsilon_{i}+2 \varepsilon_{m}}}{1-\phi \frac{\varepsilon_{i}-\varepsilon_{m}}{\varepsilon_{i}+2 \varepsilon_{m}}}\right),
$$

and

$$
=\frac{d_{h m}+\phi\left(\frac{3 \varepsilon_{m}}{\varepsilon_{i}+2 \varepsilon_{m}} d_{h i}-d_{h m}\right)\left(\frac{1+\frac{4 \mu_{m}}{3 k}}{1+\frac{4 \mu_{m}}{3 k_{i}}}\right)}{1-\phi \frac{\varepsilon_{i}-\varepsilon_{m}}{\varepsilon_{i}+2 \varepsilon_{m}}}
$$

By using Eqs. (4) and (25), the primary pyroelectric coefficient of the composite is expressed as

$$
\begin{aligned}
\rho_{1}= & \phi\left(\frac{\frac{3 \varepsilon_{m}}{\varepsilon_{i}+2 \varepsilon_{m}}}{1-\phi \frac{\varepsilon_{i}-\varepsilon_{m}}{\varepsilon_{i}+2 \varepsilon_{m}}}\right) \rho_{i} \\
& +(1-\phi)\left(\frac{1}{1-\phi \frac{\varepsilon_{i}-\varepsilon_{m}}{\varepsilon_{i}+2 \varepsilon_{m}}}\right) \rho_{m},
\end{aligned}
$$

where $\rho_{i}$ and $\rho_{m}$ denote the pyroelectric response of the inclusion and matrix, respectively. The pyroelectric contribution arising from the temperature dependence of permittivity is ignored in the present study as we consider measurement temperatures far below the Curie temperature $T_{C}$ of the two phases. However, for temperatures in the vicinity of a transition point of the phases, the temperature dependence of material properties might have significant effects on the pyroelectric activity. ${ }^{19,20}$ The secondary pyroelectricity is written as

$$
\phi \frac{\left(\frac{3 \varepsilon_{m}}{\varepsilon_{i}+2 \varepsilon_{m}} d_{h i}-d_{h m}\right)\left(\alpha-\alpha_{i}\right)\left(\frac{\frac{4 \mu_{m}}{3}}{1+\frac{4 \mu_{m}}{3 k_{i}}}\right)}{1-\phi \frac{\varepsilon_{i}-\varepsilon_{m}}{\varepsilon_{i}+2 \varepsilon_{m}}}
$$

which occurs as a result of the deformation mismatch between the two phases. Summations of Eqs. (28) and (29) give the total pyroelectric coefficient for the composite. Equations (25)-(29) are derived based on the MW approach which is believed to give expressions applicable to the dilute suspension regime.

\section{Effective-medium (EM) approach for deriving the effective constants of 0-3 pyroelectric composites in the concentrated suspension limit}

This section illustrates the derivation of expressions for the effective constants of ferroelectric 0-3 composites in the concentrated suspension regime based on an effective medium approach. ${ }^{16}$ From here onward, we skip the \langle\rangle notation in the interest of simplicity and convenience when no confusion can be caused (see Fig. 3).

Equation (D15) of Appendix D provides the EM equations as

$$
\begin{aligned}
-\frac{d \phi}{\{1-\phi\}} & =\frac{d P_{m}}{\left\{\frac{\partial P}{\partial \phi_{\phi=0}}\right\}} \\
& =\frac{d \varepsilon_{m}}{\left\{\frac{\partial \varepsilon}{\partial \phi_{\phi=0}}\right\}}=\frac{d \mu_{m}}{\left\{\frac{\partial \mu}{\partial \phi_{\phi=0}}\right\}}=\frac{d k_{m}}{\left\{\frac{\partial k}{\partial \phi_{\phi=0}}\right\}} \\
& =\frac{d \alpha_{m}}{\left\{\frac{\partial \alpha}{\partial \phi_{\phi=0}}\right\}}=\frac{d d_{h m}}{\left\{\frac{\partial d_{h}}{\partial \phi_{\phi=0}}\right\}},
\end{aligned}
$$




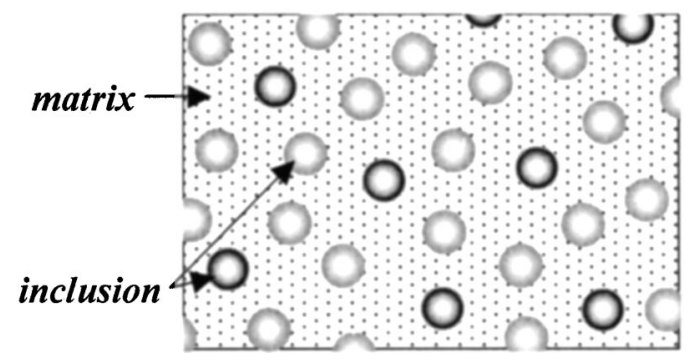

FIG. 3. Illustration of the effective-medium scheme for a composite system. Spherical inclusions are divided into two groups of "dark" and "bright" color embedded in matrix phase of effective permittivity $\varepsilon_{m}$. The darker spheres occupy a fraction $\phi_{2}$ by volume in the composites, surrounded by a "matrix" material which by itself is a composites material with a fraction of $\phi_{1}$ of the inclusion phase.

which is a set of six simultaneous equations.

The equality linking $k_{m}$ and $\alpha_{m}$ can be integrated to yield a first integral

$$
\psi_{1}=\frac{\alpha_{i}-\alpha_{m}}{\frac{1}{k_{i}}-\frac{1}{k_{m}}},
$$

which can be further manipulated to obtain a relation between $k$ and $\alpha$ of the composite as

$$
\frac{\alpha_{i}-\alpha}{\frac{1}{k_{i}}-\frac{1}{k}}=\frac{\alpha_{i}-\alpha_{m}}{\frac{1}{k_{i}}-\frac{1}{k_{m}}},
$$

which is a result identical to Eq. (23).

The equalities in Eq. (30) that link $P_{m}, d_{h m}$, and $\varepsilon_{m}$ can be combined by making use of the first integral $\psi_{1}$ to give

$$
\frac{d P_{m}-\psi_{1} \Delta T d d_{h m}}{\left\{\left(\frac{\partial P}{\partial \phi_{\phi=0}}\right)-\psi_{1} \Delta T\left(\frac{\partial d_{h}}{\partial \phi_{\phi=0}}\right)\right\}}=\frac{d \varepsilon_{m}}{\left\{3 \varepsilon_{m} \frac{\varepsilon_{i}-\varepsilon_{m}}{\varepsilon_{i}+2 \varepsilon_{m}}\right\}},
$$

where, from Appendix D,

$$
\begin{aligned}
\frac{\partial P}{\partial \phi_{\phi=0}}= & \left(P_{i}-P_{m}\right)\left(\frac{3 \varepsilon_{m}}{\varepsilon_{i}+2 \varepsilon_{m}}\right)+\left(\frac{\frac{4 \mu_{m}}{3 k_{i}}-\frac{4 \mu_{m}}{3 k_{m}}}{1+\frac{4 \mu_{m}}{3 k_{i}}}\right) \\
& \times \psi_{1} \Delta T\left(d_{h m}-d_{h i} \frac{3 \varepsilon_{m}}{\varepsilon_{i}+2 \varepsilon_{m}}\right),
\end{aligned}
$$

and

$$
\begin{array}{r}
\frac{\partial d_{h}}{\partial \phi_{\phi=0}=} d_{h m}\left(\frac{\varepsilon_{i}-\varepsilon_{m}}{\varepsilon_{i}+2 \varepsilon_{m}}-\frac{1+\frac{4 \mu_{m}}{3 k_{m}}}{1+\frac{4 \mu_{m}}{3 k_{i}}}\right) \\
+d_{h i}\left(\frac{3 \varepsilon_{m}}{\varepsilon_{i}+2 \varepsilon_{m}} \frac{1+\frac{4 \mu_{m}}{3 k_{m}}}{1+\frac{4 \mu_{m}}{3 k_{i}}}\right) .
\end{array}
$$

Manipulation of Eq. (33) yields

$$
\frac{d\left(P_{m}-\psi_{1} \Delta T d_{h m}\right)}{P_{i}-P_{m}-\psi_{1} \Delta T\left(d_{h i}-d_{h m}\right)}=\frac{d \varepsilon_{m}}{\varepsilon_{i}-\varepsilon_{m}},
$$

which may be integrated to get another first integral as

$$
\psi_{2}=\frac{P_{i}-P_{m}-\psi_{1} \Delta T\left(d_{h i}-d_{h m}\right)}{\varepsilon_{i}-\varepsilon_{m}},
$$

which then yields

$$
\frac{P_{i}-P-\psi_{1} \Delta T\left(d_{h i}-d_{h}\right)}{\varepsilon_{i}-\varepsilon}=\frac{P_{i}-P_{m}-\psi_{1} \Delta T\left(d_{h i}-d_{h m}\right)}{\varepsilon_{i}-\varepsilon_{m}},
$$

and thus the total polarization may be solved in the form

$$
\begin{aligned}
P= & P_{i}\left(\frac{\varepsilon-\varepsilon_{m}}{\varepsilon_{i}-\varepsilon_{m}}\right)+P_{m}\left(\frac{\varepsilon_{i}-\varepsilon}{\varepsilon_{i}-\varepsilon_{m}}\right)+\left(\frac{\alpha_{i}-\alpha_{m}}{\frac{1}{k_{i}}-\frac{1}{k_{m}}}\right) \\
& \times \Delta T\left(d_{h}-d_{h i} \frac{\varepsilon-\varepsilon_{m}}{\varepsilon_{i}-\varepsilon_{m}}-d_{h m} \frac{\varepsilon_{i}-\varepsilon}{\varepsilon_{i}-\varepsilon_{m}}\right) .
\end{aligned}
$$

Hence, the pyroelectric coefficients can be obtained from Eq. (36) to give the primary pyroelectric coefficient

$$
\rho_{1}=\rho_{i}\left(\frac{\varepsilon-\varepsilon_{m}}{\varepsilon_{i}-\varepsilon_{m}}\right)+\rho_{m}\left(\frac{\varepsilon_{i}-\varepsilon}{\varepsilon_{i}-\varepsilon_{m}}\right),
$$

and the secondary pyroelectric coefficient

$$
\rho_{2}=\left(\frac{\alpha_{i}-\alpha_{m}}{\frac{1}{k_{i}}-\frac{1}{k_{m}}}\right)\left(d_{h}-d_{h i} \frac{\varepsilon-\varepsilon_{m}}{\varepsilon_{i}-\varepsilon_{m}}-d_{h m} \frac{\varepsilon_{i}-\varepsilon}{\varepsilon_{i}-\varepsilon_{m}}\right) .
$$

Here, again, we have neglected the temperature dependence of the material parameters for the reason indicated in the earlier section. The effective dielectric permittivity $\varepsilon$ in Eqs. (37) and (38) can be obtained by considering the equality of Eq. (30) that relates $\varepsilon_{m}$ and $\phi$ :

$$
-\frac{d \phi}{\{1-\phi\}}=\frac{d \varepsilon_{m}}{\left\{3 \varepsilon_{m} \frac{\varepsilon_{i}-\varepsilon_{m}}{\varepsilon_{i}+2 \varepsilon_{m}}\right\}},
$$

which can be easily integrated to give

$$
\left(\frac{\varepsilon_{i}-\varepsilon}{\varepsilon^{1 / 3}}\right)=(1-\phi)\left(\frac{\varepsilon_{i}-\varepsilon_{m}}{\varepsilon_{m}^{1 / 3}}\right),
$$

a result identical to the well-known Bruggeman ${ }^{21}$ formula.

An exact expression for the effective piezoelectric coefficient $d_{h}$ is not easily obtainable from Eq. (30). However, Wong et al. ${ }^{22}$ have derived an explicit formula for $d_{h}$ as 


$$
\begin{aligned}
d_{h}= & \frac{1}{\phi}\left(\frac{\varepsilon-\varepsilon_{m}}{\varepsilon_{i}-\varepsilon_{m}}\right)\left(\frac{\frac{1}{k}-\frac{1}{k_{m}}}{\frac{1}{k_{i}}-\frac{1}{k_{m}}}\right) d_{h i}+\frac{1}{(1-\phi)}\left(\frac{\varepsilon_{i}-\varepsilon}{\varepsilon_{i}-\varepsilon_{m}}\right) \\
& \times\left(\frac{\frac{1}{k_{i}}-\frac{1}{k}}{\frac{1}{k_{i}}-\frac{1}{k_{m}}}\right) d_{h m},
\end{aligned}
$$

where $k$ is the bulk modulus as expressed in Eq. (22).

Substituting Eq. (41) and Eq. (22) into Eq. (38), the secondary pyroelectric coefficient becomes

$$
\begin{aligned}
\rho_{2}= & \left(\frac{\alpha_{i}-\alpha_{m}}{\frac{3}{4 \mu_{m}}+\frac{\phi}{k_{m}}+\frac{1-\phi}{k_{i}}}\right) \\
& \times\left(\phi \frac{\varepsilon_{i}-\varepsilon}{\varepsilon_{i}-\varepsilon_{m}} d_{h m}-(1-\phi) \frac{\varepsilon-\varepsilon_{m}}{\varepsilon_{i}-\varepsilon_{m}} d_{h i}\right),
\end{aligned}
$$

where the dielectric permittivity $\varepsilon$ is described by Eq. (40).

\section{RESULTS AND DISCUSSION}

To illustrate the pyroelectricity of ferroelectric $0-3$ composites, the biphasic system comprising ferroelectric ceramic and poly(vinylidene fluoride-trifluoroethylene) copolymer $[\mathrm{P}(\mathrm{VDF}-\mathrm{TrFE})]$ is chosen. Lead zirconate titanate (PZT) and lead titanate (PT) are the ceramic phases considered. PT is chosen as another ceramic in the present study with the purpose of comparison with PZT because the piezo and mechanical properties of PT such as piezoelectric coefficient, bulk modulus, and shear modulus are larger than those of PZT ceramic. The pyroelectricity of the 0-3 composites are investigated theoretically under various polarization configurations, particularly for the secondary pyroelectric effect. The investigations were performed theoretically for two types of composites: (i) copolymer-matrix composites (Figs. 4 and 5) and (ii) ceramic-matrix composites (Figs. 6 and 7). In practice, a composite ${ }^{23}$ system prepared by making use of "cor- als" as a template to produce a ceramic replica and then filling its pores with polymer might be a way of realizing a ceramic-matrix composite with polymer inclusions. The ceramic-matrix composite might be an interesting system to study, particularly for its secondary pyroelectric effect.

In order to illustrate and differentiate the dilute suspension (MW) and concentrated suspension (EM) models, the experimental results of PZT/P(VDF-TrFE) (Ref. 24) [matrix: $\mathrm{P}(\mathrm{VDF}-\mathrm{TrFE})$; inclusion: $\mathrm{PZT}]$ up to a high loading of 0.6 volume fraction of PZT inclusions are compared with our theoretical predictions. The physical parameters adopted in the calculations are listed in Table I. ${ }^{25}$ The Young's modulus $Y$ and Poisson's ratio $\nu$ of PT are $126.7 \mathrm{GPa}$ and 0.22 , respectively, as given by Ref. 21. Following the relation $k$ $=Y / 3(1-2 \nu)$ and $\mu=Y / 2(1+\nu)$ for the bulk modulus and shear modulus, the values for $k$ and $\mu$ are found to be 75.4 and $51.9 \mathrm{GPa}$, respectively. By using equation $d_{h}=d_{33}$ $+2 d_{31} \quad\left[d_{33}=94.0 \mathrm{pCN}^{-1}\right.$ and $d_{31}=-9.5 \mathrm{pCN}^{-1}$ (Ref. 22)], the hydrostatic piezoelectric coefficient $d_{h}$ for PT is calculated as $75.0 \mathrm{pCN}^{-1}$. The poling ratios for the constituents are for simplicity assumed to be 0.65 for both ceramic and polymer. For the composite system PZT/P(VDF-TrFE), the poling ratios are also taken as 0.65 for the convenience of discussion and comparison.

Figures 4 and 5 show the calculated total pyroelectric and secondary pyroelectric coefficient, respectively, for composite systems of PZT and PT dispersed in the P(VDFTrFE) copolymer matrix. It is found that generally the magnitudes associated with the secondary pyroelectric activity are only a small fraction of the total activity. The comparison of experimental results with our theory is given in Fig. 4(a). The EM prediction shows a good agreement with the experimental data up to a higher volume fraction of 0.6 (with most data points falling within the EM calculated lines) while the MW approach also shows a good accuracy in prediction for volume fraction up to 0.3 . This comparison shows that the effective pyroelectric coefficient derived based on the EM approach results in a better agreement with known experimental results than the MW method.
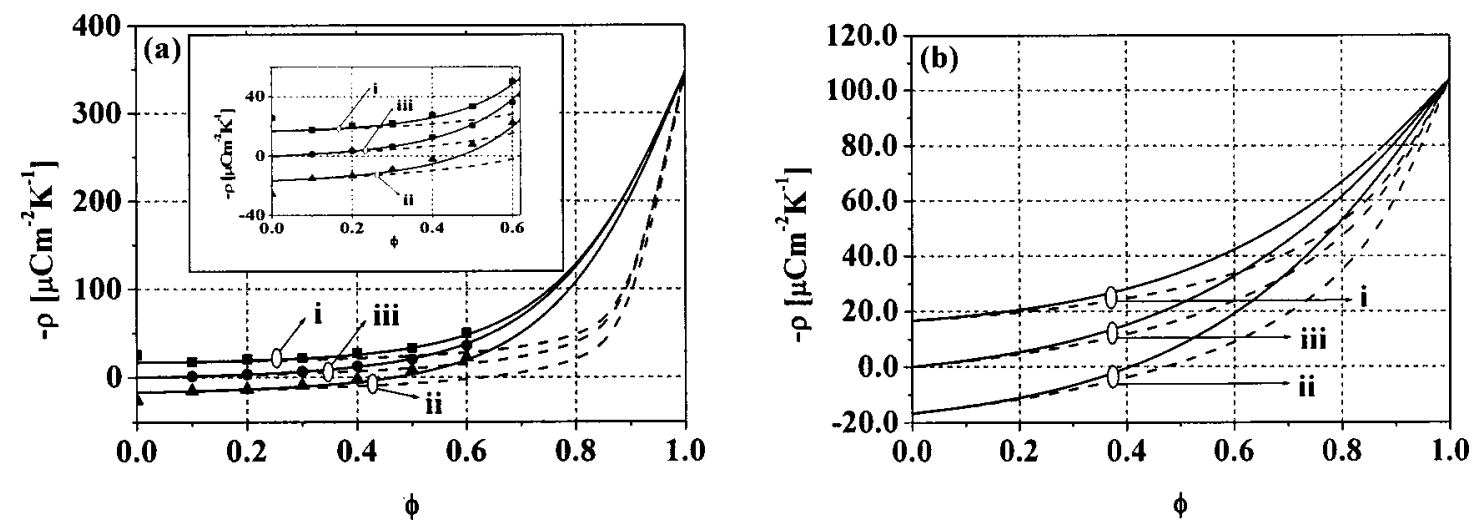

FIG. 4. Calculated total pyroelectric coefficient $-\rho$ of ferroelectric 0-3 composites with $\mathrm{P}(\mathrm{VDF}-\mathrm{TrFE})$ copolymer as matrix. Dependence of $-\rho$ on the volume fraction $\phi$ of (a) PZT and (b) PT with P(VDF-TrFE) as the matrix phase. (i) Both the matrix and inclusion constituent phases polarized in parallel; (ii) the constituent phases polarized in antiparallel directions; and (iii) only the ceramic phase is polarized. The dashed curves represent the MW predictions

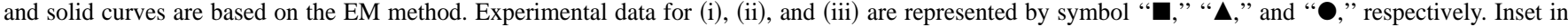
(a) shows the $-\rho$ vs $\phi$ curve in a different scale. 

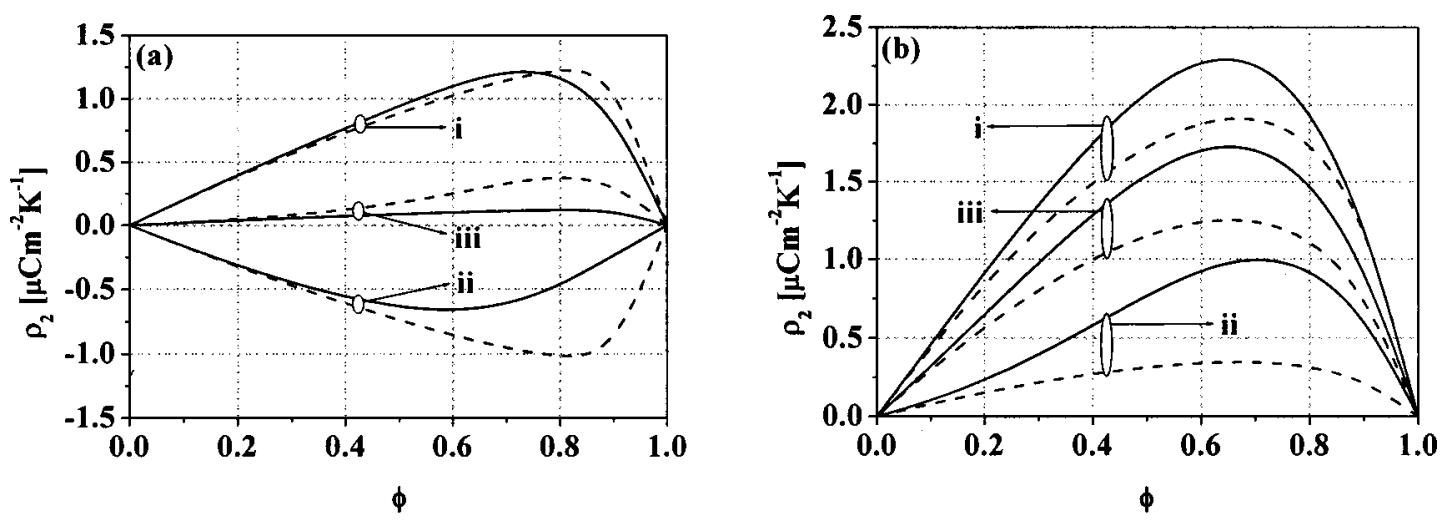

FIG. 5. Calculated secondary pyroelectric coefficient $\rho_{2}$ of ferroelectric 0-3 composites with P(VDF-TrFE) copolymer as the matrix. Dependence of $\rho_{2}$ on the volume fraction $\phi$ of (a) PZT and (b) PT with P(VDF-TrFE) as the matrix phase. (i) Both the matrix and inclusion constituent phases polarized in parallel; (ii) the constituent phases polarized in antiparallel directions; and (iii) only the ceramic phase is polarized. The dashed curves represent the MW approach predictions and solid curves are based on the EM method.

From Fig. 4, the (negative) pyroelectric coefficient of the composites increases with increasing volume fraction $\phi$, indicating the contribution of the polarized ceramic phase in all the three different polarization configurations considered: (i) the constituent phases polarized in parallel ("parallel" configuration), (ii) the constituent phases polarized in antiparallel directions ("antiparallel" configuration), and (iii) only the ceramic phase polarized ("ceramic-only" configuration). Composites polarized in the "parallel" configuration possess the highest pyroelectricity for all ceramic volume fraction. For PZT/P(VDF-TrFE) composites, the EM prediction shows that for the three configurations the total $-\rho$ increases gradually up to volume fraction of 0.6 and thereafter increases significantly with increasing $\phi$. However, the calculations from the MW method indicate a rapid enhancement of $-\rho$ only beyond $\phi=0.8$. For the PT/P(VDF-TrFE) composites shown in Fig. 4(b), both models also predict a monotonic rise in the total $-\rho$ value throughout the whole ceramic volume fraction range. An interesting feature that can be observed in both PZT and PT composite systems for the "antiparallel" configuration is that the magnitude of the pyroelectric activity $\rho$ is suppressed gradually with increasing volume fraction of ceramic inclusions. Until the pyroactivity totally vanishes at a "critical" nonzero volume fraction $\left[\phi_{C} \approx 0.5(\mathrm{PZT})\right.$ and $\phi_{C} \approx 0.4(\mathrm{PT})$ for $\mathrm{EM} ; \phi_{C} \approx 0.6(\mathrm{PZT})$ and $\phi_{C} \approx 0.5$ (PT) for MW] before enhancing with further increase in $\phi$. It is noted that PZT/P(VDF-TrFE) loses its pyroelectricity at higher ceramic concentration $\phi$ than PT/ $\mathrm{P}(\mathrm{VDF}-\mathrm{TrFE})$ composites, while the hand-waiving one would be tempted to believe the opposite because it would take more PT particles (of lower pyroelectric coefficient than PZT) to counteract the effect of the copolymer matrix.

In general, Fig. 5 shows that the contributions of piezoelectrically induced charges in the two composite systems are not significant compared to the charges produced due to variation of polarization with temperature. The secondary pyroelectric activity in the $\mathrm{PT} / \mathrm{P}(\mathrm{VDF}-\mathrm{TrFE})$ composites is slightly larger than that of the PZT/P(VDF-TrFE) system for all three polarization configurations. For the two systems, the secondary pyroelectric response reaches its peak at ceramic volume fraction greater than 0.6. Among the three polarization configurations, the secondary responses of the "parallel" and "ceramic-only" configurations for the two composite systems show a similar trend.

Figures 6 and 7 show the pyroelectric responses of composite systems of $\mathrm{P}(\mathrm{VDF}-\mathrm{TrFE})$ inclusions in the ferroelec-
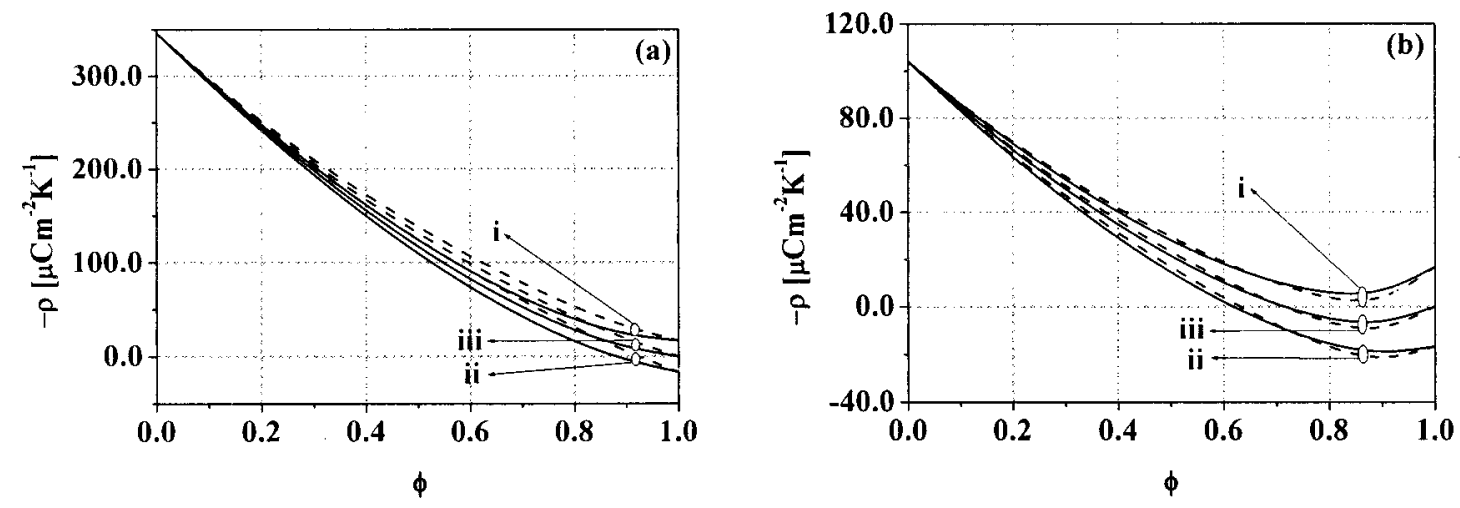

FIG. 6. Calculated total pyroelectric coefficient $-\rho$ of ferroelectric $0-3$ composites with ceramic as the matrix. Dependence of $-\rho$ on the volume fraction $\phi$ of P(VDF-TrFE) with (a) PZT and (b) PT as the matrix phase. (i) Both the matrix and inclusion constituent phases polarized in parallel; (ii) the constituent phases polarized in antiparallel directions; and (iii) only the ceramic phase is polarized. The dashed curves represent the MW predictions and solid curves are based on the EM method. 

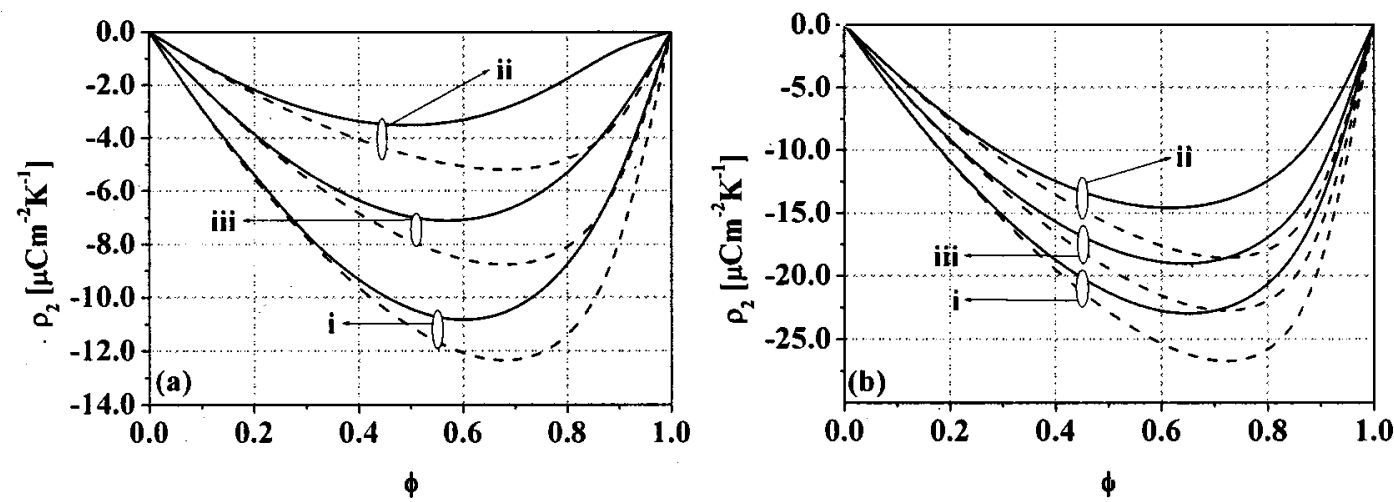

FIG. 7. Calculated secondary pyroelectric coefficient $\rho_{2}$ of ferroelectric 0-3 composites with copolymer as the matrix. Dependence of $\rho_{2}$ on the volume fraction $\phi$ of $\mathrm{P}(\mathrm{VDF}-\mathrm{TrFE})$ with (a) PZT and (b) PT as the matrix phase. (i) Both the matrix and inclusion constituent phases polarized in parallel; (ii) the constituent phases polarized in antiparallel directions; and (iii) only the ceramic phase is polarized. The dash curves represent the MW predictions and solid curves are based on the EM method.

tric ceramic matrix. The predictions show that the pyroelectric response decreases monotonically with increasing $\phi$, indicating a weakening of pyroelectric activity as the concentration of copolymer inclusions is higher, which is expected as the pyroelectricity of the copolymer is weaker than that of the two ceramics. An interesting feature is shown in Fig. 6 by the "antiparallel" polarization configuration in which the pyroelectric response declines with increasing copolymer volume fraction and vanishes at some critical concentration $\phi_{C}$. The elimination of pyroelectricity in the composite systems near $\phi_{C}$ means the strengths of the primary and secondary effects are similar, but with opposite signs. However, a higher volume fraction of copolymer inclusions is required to cancel the pyroelectricity of the PZT-matrix compared to PT-matrix composite system. This is because the pyroelectric response of PZT is much stronger than that of PT. Upon further increasing the volume fraction of copolymer inclusions, the pyroelectric activity of the PT-matrix composite is increased in an opposite direction and ap-

TABLE I. Physical parameters for P(VDF-TrFE), PZT, and PT phases adopted in the numerical calculations for the composites as shown in Figs. $4-7$.

\begin{tabular}{cccc}
\hline \hline & $\mathrm{P}(\mathrm{DVF}-\mathrm{TrFE})^{\mathrm{a}}$ & $\mathrm{PZT}^{\mathrm{a}}$ & $\mathrm{PT}^{\mathrm{b}, \mathrm{c}, \mathrm{d}}$ \\
\hline $\begin{array}{c}\rho^{\mathrm{e}} \\
\left(\mu \mathrm{Cm}^{-2} \mathrm{~K}^{-1}\right)\end{array}$ & -16.71 & -346.45 & $-104.00^{\mathrm{b}}$ \\
$k$ \\
$(\mathrm{GPa})$ & 3.42 & 62.30 & $75.42^{\mathrm{c}}$ \\
$\mu$ & & & \\
$(\mathrm{GPa})$ & 0.73 & 27.10 & $51.93^{\mathrm{c}}$ \\
$\alpha^{\mathrm{f}}$ & 74.50 & 3.90 & $3.57^{\mathrm{d}}$ \\
$\left(\times 10^{-6} \mathrm{~K}^{-1}\right)$ & -9.30 & 34.00 & $75.00^{\mathrm{c}}$ \\
$d_{h}$ & $9.50^{\mathrm{g}}$ & 1116.00 & $140.00^{\mathrm{b}}$ \\
$\left(\mathrm{pCN}^{-1}\right)$ & & & \\
$\varepsilon$ & &
\end{tabular}

${ }^{a}$ Reference 24.

${ }^{\mathrm{b}}$ Reference 19 .

${ }^{\mathrm{c}}$ Reference 22.

${ }^{\mathrm{d}}$ Reference 25.

${ }^{\mathrm{e}}$ The values are adopted by assuming a poling ratio of 0.65 .

${ }^{\mathrm{f}}$ Linear thermal expansion coefficient.

${ }^{\mathrm{g}}$ The value for unpolarized state is $\varepsilon=11.2$. proaches a minimum around $\phi=0.85$ [Fig. 6(b)] before turning back. A similar trend is also observed for the ceramiconly polarization configuration, as shown in Fig. 6(b).

Compared to the copolymer-matrix composites in Fig. 5(b), the secondary pyroelectric response for ceramic-matrix composite systems exhibits negative values in all the three polarization configurations. The secondary pyroelectric activity is stronger than that of the conventional copolymermatrix systems. A feature noted in Fig. 7 is that the secondary pyroelectric activity of the PT-matrix composite system dominates at a certain range of copolymer volume fraction. This clearly demonstrates that the charge induced due to piezoelectricity resulting from thermal deformation is stronger than that arising from polarization changes at that particular concentration region.

\section{CONCLUSION}

Analytical expressions for the pyroelectric activities of ferroelectric 0-3 composites have been developed using two different methods: the MW approach and the EM method. 0-3 composites consisting of ferroelectric ceramics and $\mathrm{P}(\mathrm{VDF}-\mathrm{TrFE})$ are adopted for the present study. For the ceramic phases, PZT and PT are considered in this investigation owing to their difference in piezo and mechanical properties, which are the important factors for secondary pyroelectric effects. The experimental results for the copolymer-matrix composites of PZT/P(VDF-TrFE) (Ref. 24) are used to compare with the calculation results from the two theoretical approaches. The predictions from EM show a better agreement with experimental data up to higher volume fraction of inclusion than that from the MW model.

Two different kinds of 0-3 composites have been examined theoretically: (a) copolymer-matrix composites and (b) ceramic-matrix composites. The copolymer-matrix composite is the conventional composite system comprising copolymer and ceramic as matrix and inclusion, respectively. Ceramic-matrix composites are a special type of composites consisting of ceramics as matrix phase and copolymer as inclusions. For copolymer-matrix composites, the results show that PZT-inclusion composites (i.e., composites with 
PZT inclusions) exhibit a higher pyroelectric effect than copolymer-matrix composites with PT as inclusions. Compared to PZT-inclusion composites, the secondary pyroelectric coefficient for PT-inclusion composites is larger. This is expected as the piezo and mechanical properties of PT are higher than those of PZT ceramic. In general, the results show that the secondary effect is only a small fraction of the total pyroelectric effect in the copolymer-matrix composites. This indicates that the pyroelectric activities in copolymermatrix composites are dominated by the primary pyroactivities. In ceramic-matrix composites, the results show that the secondary effect is larger than the copolymer-matrix composites. Although the secondary pyroelectric coefficient of the composites can be enhanced in ceramic-matrix geometry, the composites (the ceramic-matric composites) still exhibit a dominant primary pyroactivity, similar to the copolymermatrix composites.

More interesting results can be found by examining the pyroelectric responses of the ferroelectric 0-3 composites under three different polarization configurations, particularly with the constituent phases poled in antiparallel directions. For the "antiparallel" configuration, the piezoelectricities of the two constituent phases are enhanced whereas the pyroelectric activities are partially eliminated. For instance, the contributions of the two constituent phases (ceramic and copolymer) to the effective pyroelectric coefficient for the copolymer-matrix composite are neutralized at a "critical" nonzero volume fraction of $\phi_{C} \approx 0.4$ for PT or $\phi_{C} \approx 0.5$ for PZT (based on the predictions from the EM method). Pyroelectric-compensated piezoelectric composite materials are predicted to occur at a lower volume fraction of inclusions for copolymer-matrix composites than for ceramicmatrix composites.

In this study, we are concerned only with the pyroelectric response at a temperature well below the Curie temperature of the two constituents. At temperatures near the transition temperature of $\mathrm{P}(\mathrm{VDF}-\mathrm{TrFE}),{ }^{19,20}$ its dielectric permittivity exhibits a strong dependence on temperature. ${ }^{19,20}$ A detailed theoretical study on the pyroelectric effect by taking into account the temperature dependence of dielectric permittivity will be performed in a later publication.

\section{ACKNOWLEDGMENT}

This work was supported by the Centre for Smart Materials and an internal research grant of The Hong Kong Polytechnic University.

\section{APPENDIX A}

Consider a system comprising a single dielectric sphere of radius $R$ in an infinite ferroelectric matrix under an applied field in the $z$ direction. We assume that there are no surface charges on the sphere and that hydrostatic stresses may be present in the constituents. We write the electric displacement $D$ of the inclusion $(i)$ and matrix $(m)$ as $D_{i}=P_{i}$ $+\varepsilon_{i} E_{i}+d_{h i} \sigma_{i}$ and $D_{m}=P_{m}+\varepsilon_{m} E_{m}+d_{h m} \sigma_{m}$ where the polarizations $P$ and stresses $\sigma$ are assumed uniform, which may be denoted by their average values \langle\rangle$. E, \varepsilon$, and $d_{h}$ denote the electric field, permittivity, and hydrostatic piezoelectric coefficients. Under these simplifying assumptions, we need to solve the Laplace's equation

$$
\nabla^{2} \varphi_{l}(r, \theta)=0, \quad l=i, m,
$$

where $\varphi_{i}(r, \theta)$ is the electric potential for $r<R$ inside the spherical inclusion and $\varphi_{m}(r, \theta)$ is the potential for $r>R$ outside of the inclusion. Coordinates $r$ and $\theta$ represent the distance from the center of the sphere and azimuthal angle, respectively, $\theta=0$ being the $z$ axis.

The Laplace's equations are subject to the following conditions:

$$
\text { at } r=0, \quad \varphi_{i}(0, \theta) \text { is finite; }
$$

(ii) by assuming the field in the matrix sufficiently far away from the inclusion is uniform and denoted by $E_{m \infty}$ at

$r \rightarrow \infty, \quad \varphi_{m}(r \rightarrow \infty, \theta)=-E_{m \infty} r \cos \theta$;

(iii) at $r=R$, the boundary conditions for the continuity of the electric potential is

$\varphi_{i}(R, \theta)=\varphi_{m}(R, \theta)$,

and for the normal component of the electric displacement is

$$
\begin{aligned}
& -\varepsilon_{i} \frac{\partial \varphi_{i}}{\partial r}+\left\langle P_{i}\right\rangle \cos \theta+d_{h i}\left\langle\sigma_{i}\right\rangle \cos \theta \\
& =-\varepsilon_{m} \frac{\partial \varphi_{m}}{\partial r}+\left\langle P_{m}\right\rangle \cos \theta+d_{h m}\left\langle\sigma_{m}\right\rangle \cos \theta .
\end{aligned}
$$

From Eq. (A1) together with the conditions of Eqs. (A2) and (A3), the general solutions for the spherical inclusion and matrix are

$$
\varphi_{i}(r, \theta)=\sum_{n=0}^{\infty} A_{n} r^{n} \rho_{n}(\cos \theta),
$$

and

$$
\varphi_{m}(r, \theta)=-E_{m \infty} r \cos \theta+\sum_{n=0}^{\infty} \frac{C_{n}}{r^{n+1}} \rho_{n}(\cos \theta),
$$

where $\rho_{n}(\cos \theta)$ are Legendre polynomials.

Using Eqs. (A6) and (A7) together with boundary conditions (A4) and (A5), we obtain

$$
\begin{aligned}
A_{1}= & -\left(\frac{3 \varepsilon_{m}}{\varepsilon_{i}+2 \varepsilon_{m}}\right) E_{m \infty} \\
& +\frac{\left\langle P_{i}\right\rangle-\left\langle P_{m}\right\rangle+d_{h i}\left\langle\sigma_{i}\right\rangle-d_{h m}\left\langle\sigma_{m}\right\rangle}{\varepsilon_{i}+2 \varepsilon_{m}}, \\
C_{1}= & R^{3}\left\{\left(\frac{\varepsilon_{i}-\varepsilon_{m}}{\varepsilon_{i}+2 \varepsilon_{m}}\right) E_{m \infty}\right. \\
& \left.+\frac{\left\langle P_{i}\right\rangle-\left\langle P_{m}\right\rangle+d_{h i}\left\langle\sigma_{i}\right\rangle-d_{h m}\left\langle\sigma_{m}\right\rangle}{\varepsilon_{i}+2 \varepsilon_{m}}\right\},
\end{aligned}
$$

and 


$$
A_{n}=C_{n}=0 \text { for } n \neq 1 \text {. }
$$

Substituting Eqs. (A8)-(A10) into Eq. (A6), the electric field in the inclusion is found to be independent of $(r, \theta)$, thus $\left\langle E_{i}\right\rangle=E_{i} . E_{m \infty}$ is likewise obtained from Eq. (A7), which readily verifies that $\left\langle E_{m}\right\rangle=E_{m \infty}$ by averaging over a spherical matrix volume $r>R$ outside the inclusion. The expression for the field in the inclusion is thus

$$
\left\langle E_{i}\right\rangle=\frac{\left(\varepsilon_{m}\left\langle E_{m}\right\rangle+\left\langle P_{m}\right\rangle+d_{h m}\left\langle\sigma_{m}\right\rangle\right)-\left(\left\langle P_{i}\right\rangle+d_{h i}\left\langle\sigma_{i}\right\rangle\right)+2 \varepsilon_{m}\left\langle E_{m}\right\rangle}{\varepsilon_{i}+2 \varepsilon_{m}},
$$

which can be rewritten in terms of the average dielectric displacement and electric field as

$$
\left\langle D_{i}\right\rangle+2 \varepsilon_{m}\left(\left\langle E_{i}\right\rangle-\left\langle E_{m}\right\rangle\right)=\left\langle D_{m}\right\rangle,
$$

where

$$
\left\langle D_{i}\right\rangle=\varepsilon_{i}\left\langle E_{i}\right\rangle+\left\langle P_{i}\right\rangle+d_{h i}\left\langle\sigma_{i}\right\rangle,
$$

and

$$
\left\langle D_{m}\right\rangle=\varepsilon_{m}\left\langle E_{m}\right\rangle+\left\langle P_{m}\right\rangle+d_{h m}\left\langle\sigma_{m}\right\rangle .
$$

\section{APPENDIX B}

Consider a spherical inclusion of radius $r=a$ embedded in a continuous matrix. The center of the sphere is chosen as the origin of spherical coordinates $(r, \theta, \phi)$. Let us consider the system subjected to hydrostatic stresses. Hydrostatic stresses are independent of $\theta$ and $\phi$, thus shear stresses vanish and $\sigma_{\theta \theta}=\sigma_{\phi \phi}$. The equilibrium equation is

$$
\frac{\partial \sigma_{r r}}{\partial r}+\frac{2\left(\sigma_{r r}-\sigma_{\theta \theta}\right)}{r}=0,
$$

and the displacement equation corresponding to stress (B1) is in the form

$$
\frac{\partial^{2} u_{r r}}{\partial r^{2}}+\frac{2}{r} \frac{\partial u_{r r}}{\partial r}-\frac{2}{r^{2}} u_{r r}=0 .
$$

Rearranging the above equation results in

$$
\frac{\partial}{\partial r}\left[\frac{\partial u_{r r}}{\partial r}+\frac{2}{r} u_{r r}\right]=0,
$$

which can be solved by successive integration to give

$$
u_{r r}=A r+\frac{B}{r^{2}},
$$

where $A$ and $B$ are integration constants.

Equation (B3) gives the general solutions for the inclusion and matrix as

$$
u_{r r i}=A_{i} r,
$$

and

$$
u_{r r m}=A_{m} r+\frac{B_{m}}{r^{2}},
$$

where subscripts $i$ and $m$ represent inclusion and matrix, respectively. The coefficient $B_{i}$ for the inclusion vanishes because $u_{r r i}(0, \theta, \phi)$ is finite at $r=0$.
The strains of the inclusion and matrix can be obtained from the displacement Eqs. (B4) and (B5) as

$$
e_{r r i}=A_{i},
$$

and

$$
e_{r r m}=A_{m}+\frac{B_{m}}{r^{3}} .
$$

The corresponding radial stresses for the inclusion and matrix are found to be

$$
\sigma_{r r i}=3 k_{i} A_{i},
$$

and

$$
\sigma_{r r m}=3 k_{m} A_{m}-4 \mu_{m} \frac{B_{m}}{r^{3}},
$$

where $k$ and $\mu$ are bulk modulus and shear modulus, respectively.

The constants of integration are subject to boundary conditions as below:

$$
\text { at } r=a, u_{r i}=u_{r m} \text {, }
$$

and

$$
\sigma_{r r i}=\sigma_{r r m} .
$$

Equations (B4) and (B5) together with boundary conditions (B10) give

$$
A_{i}=A_{m}+\frac{B_{m}}{a^{3}},
$$

and Eqs. (B8) and (B9) together with boundary conditions (B10) give

$$
3 k_{i} A_{i}=3 k_{m} A_{m}-4 \mu_{m} \frac{B_{m}}{a^{3}} .
$$

Solving Eqs. (B11) and (B12) yields

$$
3 k_{m} A_{m}=3 k_{i} A_{i}-4 \mu_{m}\left(A_{i}-A_{m}\right) \text {. }
$$

The stress-strain relationship can be obtained from Eqs. (B6) to (B9) as

$$
\left\langle\sigma_{m}\right\rangle=\left\langle\sigma_{i}\right\rangle+\frac{4}{3} \mu_{m}\left(\left\langle e_{i}\right\rangle-\left\langle e_{m}\right\rangle\right),
$$

where the volumetric strains for matrix and inclusion are $\left\langle e_{i}\right\rangle=3 A_{i}$ and $\left\langle e_{m}\right\rangle=3 A_{m}$. 


\section{APPENDIX C}

Consider the following manipulations in which $\langle D\rangle$, $\langle E\rangle$, and $\langle\sigma\rangle$ are the volume averaged dielectric displacement, electric field, and hydrostatic stress, respectively; symbols with subscripts $i$ and $m$ are associated with inclusion and matrix, respectively, and those without usually refer to the composite. We first consider the volume-averaged quantity

$$
\begin{aligned}
I_{1} & \equiv\left\langle D-P_{m}-\varepsilon_{m} E-d_{h m}\right\rangle \\
& =\langle D\rangle-\left\langle P_{m}\right\rangle-\varepsilon_{m}\langle E\rangle-d_{h m}\langle\sigma\rangle .
\end{aligned}
$$

Since $P_{m}$ has been assumed uniform, it is the same as $\left\langle P_{m}\right\rangle$. $I_{1}$ can also be expressed in terms of the volume of the composite $V$ and of inclusion $V_{i}$ as

$$
\begin{aligned}
I_{1} & =\frac{1}{V} \int_{V}\left(D-P_{m}-\varepsilon_{m} E-d_{h m} \sigma\right) d V, \\
& =\frac{1}{V} \int_{V_{i}}\left(D-P_{m}-\varepsilon_{m} E-d_{h m} \sigma\right) d V, \\
& =\frac{V_{i}}{V}\left(\left\langle D_{i}\right\rangle-\left\langle P_{m}\right\rangle-\varepsilon_{m}\left\langle E_{i}\right\rangle-d_{h m}\left\langle\sigma_{i}\right\rangle\right),
\end{aligned}
$$

where it is noted that the integral over the matrix volume is zero because $\left\langle D_{m}\right\rangle=\left\langle P_{m}\right\rangle+\varepsilon_{m}\left\langle E_{m}\right\rangle+d_{h m}\left\langle\sigma_{m}\right\rangle$.

Equation $(\mathrm{C} 1)$ then yields

$$
\begin{aligned}
& \langle D\rangle-\left\langle P_{m}\right\rangle-\varepsilon_{m}\langle E\rangle-d_{h m}\langle\sigma\rangle \\
& \quad=\phi\left(\left\langle D_{i}\right\rangle-\left\langle P_{m}\right\rangle-\varepsilon_{m}\left\langle E_{i}\right\rangle-d_{h m}\left\langle\sigma_{i}\right\rangle\right),
\end{aligned}
$$

where $\phi \equiv V_{i} / V$ is the volume fraction of the inclusion phase.

Similarly, the volume-averaged quantity

$$
I_{2} \equiv\left\langle\sigma-k_{m} e-k_{m} \alpha_{m} \Delta T\right\rangle=\langle\sigma\rangle-k_{m}\langle e\rangle+k_{m} \alpha_{m} \Delta T,
$$

may be expressed as

$$
\begin{aligned}
I_{2} & =\frac{1}{V} \int_{V}\left(\sigma-k_{m} e+k_{m} \alpha_{m} \Delta T\right) d V, \\
& =\frac{1}{V} \int_{V_{i}}\left(\sigma-k_{m} e+k_{m} \alpha_{m} \Delta T\right) d V, \\
& =\frac{V_{i}}{V}\left(\left\langle\sigma_{i}\right\rangle-k_{m}\left\langle e_{i}\right\rangle+k_{m} \alpha_{m} \Delta T\right),
\end{aligned}
$$

where, again, it is noted that the integration over the matrix volume gives zero as $\left\langle\sigma_{m}\right\rangle=k_{m}\langle e\rangle+k_{m} \alpha_{m} \Delta T$. Then, Eqs. (C4) and (C5) yield

$$
\langle\sigma\rangle-k_{m}\langle e\rangle+k_{m} \alpha_{m} \Delta T=\phi\left(\left\langle\sigma_{i}\right\rangle-k_{m}\left\langle e_{i}\right\rangle+k_{m} \alpha_{m} \Delta T\right) .
$$

\section{APPENDIX D}

We now consider a composite system consisting of inclusions with total volume fraction $\phi$ in a matrix material. For the convenience of illustration, the inclusions are divided into two groups, which are represented in Fig. 3 as bright and dark spheres. The dark spheres occupy (say) a fraction $\phi_{2}$ of the composite and they are dispersed in a "matrix" environment, which is itself a composite material made up of bright spheres of (say) fraction $\phi_{1}$ in pure matrix materials. The total volume fraction $\phi$ of the inclusions expressed in terms of $\phi_{1}$ and $\phi_{2}$ is

$$
\phi=\left(1-\phi_{2}\right) \phi_{1}+\phi_{2} \text {. }
$$

Let the formulas for calculating the overall or effective polarization, dielectric permittivity, piezoelectric coefficient, bulk modulus, shear modulus, and thermal expansion coefficient of the composite be represented by functions of the constituent properties and the inclusion fraction as below:

$P=P\left(P_{i}, P_{m}, \varepsilon_{i}, \varepsilon_{m}, k_{i}, k_{m}, \mu_{i}, \mu_{m}, \alpha_{i}, \alpha_{m}, d_{h i}, d_{h m}, \phi\right)$,

$\varepsilon=\varepsilon\left(\varepsilon_{i}, \varepsilon_{m}, \phi\right)$,

$d_{h}=d_{h}\left(\varepsilon_{i}, \varepsilon_{m}, k_{i}, k_{m}, \mu_{i}, \mu_{m}, d_{h i}, d_{h m}, \phi\right)$,

$k=k\left(k_{i}, k_{m}, \mu_{i}, \mu_{m}, \phi\right)$,

$\mu=\mu\left(k_{i}, k_{m}, \mu_{i}, \mu_{m}, \phi\right)$,

and

$$
\alpha=\alpha\left(k_{i}, k_{m}, \alpha_{i}, \alpha_{m}, \phi\right),
$$

where the functions $P, \varepsilon, d_{h}, k, \mu$, and $\alpha$ are to be determined by the EM approach. In writing down these equations, the relevant independent variables are decided by referring to Eqs. (22), (23), (25), (26), and (27) for the dilute suspension case. We refer, e.g., to the Hashin ${ }^{16}$ shear modulus

$$
\begin{aligned}
\mu= & \mu_{m}\{1 \\
& \left.+\frac{15\left(1-\nu_{m}\right)\left(\mu_{i} / \mu_{m}-1\right) \phi}{7-5 \nu_{m}+2\left(4-5 \nu_{m}\right)\left[\mu_{i} / \mu_{m}-\left(\mu_{i} / \mu_{m}-1\right) \phi\right]}\right\},
\end{aligned}
$$

where $\nu_{m}$ is Poisson's ratio of the matrix phase, to obtain the functional dependence as expressed in Eq. (D6). Thus, e.g., $\alpha$ of the composite is calculated from the knowledge of $k$ and $\alpha$ of the constituents.

First, we wish to simplify the rather clumpsy notation in Eqs. (D1)-(D7). Let $\Theta$ represent an array of properties for the composite material system and $\Theta^{n}$ its individual elements. The symbol $\Theta^{n}$ for $n=1,2,3,4,5$, and 6 correspond to $P, \varepsilon, d_{h}, k, \mu$, and $\alpha$, respectively. When $\Theta$ is written with a subscript $\ell$ as $\Theta_{\ell}$, then $\ell$ labels the specific system or material in question. Thus, we denote by $\Theta_{i}$ and $\Theta_{m}$ the arrays of properties of the inclusion material and matrix material, respectively. Thus, Eqs. (D2)-(D7) can be abridged as

$$
\Theta^{n}=\Theta^{n}\left(\Theta_{i}, \Theta_{m}, \phi\right) \text { for } n=1,2, \ldots, 6 \text {, }
$$

or simply as $\Theta=\Theta\left(\Theta_{i}, \Theta_{m}, \phi\right)$, which can be written in terms of $\phi_{1}$ and $\phi_{2}$ by using Eq. (D1) as

$$
\Theta=\Theta\left[\Theta_{i}, \Theta_{m},\left(1-\phi_{2}\right) \phi_{1}+\phi_{2}\right] \text {. }
$$

Now back to the main discussion. An effective-medium scheme can be developed by considering that the dark inclusions of volume fraction $\phi_{2}$ in the composite (Fig. 3) are embedded in an "effective medium" (composite with inclusion fraction $\phi_{1}$ ) having effective properties $P_{1}, \varepsilon_{1}, d_{h 1}$, 
$k_{1}, \mu_{1}$, and $\alpha_{1}$. According to the notation above, we denote the array of properties of the latter composite by $\Theta_{1}$ and that of the former composite by $\Theta_{2}$. Thus, the effective properties of the original composite can be calculated as

$$
\Theta_{2}=\Theta\left(\Theta_{i}, \Theta_{1}, \phi_{2}\right) \text {. }
$$

Now

$$
\Theta_{1}=\Theta\left(\Theta_{i}, \Theta_{m}, \phi_{1}\right) \text {. }
$$

By utilizing Eq. (D11), Eq. (D10) can be reexpressed as

$$
\Theta_{2}=\Theta\left[\Theta_{i}, \Theta\left(\Theta_{i}, \Theta_{m}, \phi_{1}\right), \phi_{2}\right] .
$$

However, all the time we have been looking at the same composite system we started with (Fig. 3), only from different considerations. The assertion that the different considerations should give the same results is the statement on which the EM scheme of calculations is based. Thus, we have

$$
\begin{aligned}
& \Theta\left[\Theta_{i}, \Theta_{m},\left(1-\phi_{2}\right) \phi_{1}+\phi_{2}\right] \\
& \quad=\Theta\left[\Theta_{i}, \Theta\left(\Theta_{i}, \Theta_{m}, \phi_{1}\right), \phi_{2}\right] .
\end{aligned}
$$

Differentiation of Eqs. (D13) with respect to $\phi_{1}$ and then evaluating the results at $\phi_{1}=0$ yield six equations of similar structure, the first one $\left(\Theta^{1}\right.$ or $\left.P\right)$ of which is listed below:

$$
-\frac{\partial \Theta^{1}}{\partial \phi}\{1-\phi\}+\sum_{n} \frac{\partial \Theta^{1}}{\partial \Theta_{m}^{n}} \frac{\partial \Theta^{n}}{\partial \phi}=0,
$$

where use has been made of the equalities

$$
\Theta\left(\Theta_{i}, \Theta_{m}, 0\right)=\Theta_{m},
$$

describing the evident fact that the properties of a "composite" with zero fraction of inclusion material are just the properties of the matrix material.

Written in a more usual way, Eq. (D14) is a first-order partial differential equation of the form

$$
\begin{aligned}
-\{1 & -\phi\} \frac{\partial P}{\partial \phi}+\frac{\partial P}{\partial \phi_{\phi=0}} \frac{\partial P}{\partial P_{m}}+\frac{\partial \varepsilon}{\partial \phi} \phi_{\phi=0} \frac{\partial P}{\partial \varepsilon_{m}} \\
+ & \frac{\partial k}{\partial \phi_{\phi=0}} \frac{\partial P}{\partial k_{m}}+\frac{\partial \mu}{\partial \phi_{\phi=0}} \frac{\partial P}{\partial \mu_{m}}+\frac{\partial \alpha}{\partial \phi_{\phi=0}} \frac{\partial P}{\partial \alpha_{m}} \\
+ & \frac{\partial d_{h}}{\partial \phi_{\phi=0}} \frac{\partial P}{\partial d_{h m}}=0,
\end{aligned}
$$

where $P$ is the unknown function to be solved whereas the derivatives at $\phi=0$ are known functions pertaining to the dilute limit, which may be calculated from Eqs. (22), (23), (25), (26), and (27) and the adopted Hashin's ${ }^{16}$ shear modulus (as mentioned earlier). Thus,

$$
\begin{aligned}
\frac{\partial P}{\partial \phi_{\phi=0}}= & \left\{\left(P_{i}-P_{m}\right)\left(\frac{3 \varepsilon_{m}}{\varepsilon_{i}+2 \varepsilon_{m}}\right)+\left(\frac{4 \mu_{m} / 3}{1+4 \mu_{m} / 3 k_{i}}\right)\right. \\
& \left.\times\left(\alpha_{i}-\alpha_{m}\right) \Delta T\left(d_{h m}-d_{h i} \frac{3 \varepsilon_{m}}{\varepsilon_{i}+2 \varepsilon_{m}}\right)\right\},
\end{aligned}
$$

$$
\begin{aligned}
& \frac{\partial \varepsilon}{\partial \phi_{\phi=0}}=\left\{3 \varepsilon_{m} \frac{\varepsilon_{i}-\varepsilon_{m}}{\varepsilon_{i}+2 \varepsilon_{m}}\right\}, \\
& \frac{\partial k}{\partial \phi_{\phi=0}}=\left\{\frac{k_{m}}{k_{i}}\left(k_{i}-k_{m}\right)\left(\frac{1+4 \mu_{m} / 3 k_{m}}{1+4 \mu_{m} / 3 k_{i}}\right)\right\}, \\
& \frac{\partial \mu}{\partial \phi_{\phi=0}}=\left\{\frac{15\left(1-\nu_{m}\right)\left(\mu_{i}-\mu_{m}\right) \mu_{m}}{\left(7-5 \nu_{m}\right) \mu_{m}+2\left(4-5 \nu_{m}\right) \mu_{i}}\right\}, \\
& \frac{\partial \alpha}{\partial \phi_{\phi=0}}=\left\{\left(\alpha_{i}-\alpha_{m}\right)\left(\frac{1+4 \mu_{m} / 3 k_{m}}{1+4 \mu_{m} / 3 k_{i}}\right)\right\},
\end{aligned}
$$

and

$$
\begin{aligned}
\frac{\partial d_{h}}{\partial \phi_{\phi=0}=} & \left\{d_{h m}\left(-\frac{1+4 \mu_{m} / 3 k_{m}}{1+4 \mu_{m} / 3 k_{i}}+\frac{\varepsilon_{i}-\varepsilon_{m}}{\varepsilon_{i}+2 \varepsilon_{m}}\right)\right. \\
& \left.+d_{h i}\left(\frac{3 \varepsilon_{m}}{\varepsilon_{i}+2 \varepsilon_{m}}\right)\left(\frac{1+4 \mu_{m} / 3 k_{m}}{1+4 \mu_{m} / 3 k_{i}}\right)\right\} .
\end{aligned}
$$

${ }^{1}$ P. Muralt, Rep. Prog. Phys. 64, 1339 (2001).

${ }^{2}$ R. Watton, Ferroelectrics 91, 87 (1989).

${ }^{3}$ A. Seifert, L. Sagalowicz, P. Muralt, and N. Setter, Appl. Phys. Lett. 72, 2409 (1998)

${ }^{4}$ R. E. Newnham, D. P. Skinner, and L. E. Cross, Mater. Res. Bull. 13, 525 (1978).

${ }^{5}$ A. S. Bhalla, R. E. Newnham, L. E. Cross, W. A. Schulze, J. P. Dougherty, and W. A. Smith, Ferroelectrics 33, 139 (1981).

${ }^{6}$ H. L. W. Chan, P. K. L. Ng, and C. L. Choy, Appl. Phys. Lett. 74, 3031 (1999)

${ }^{7}$ C. J. Dias and D. K. Das-Gupta, Key Engineering Materials: Ferroelectric Polymers and Ceramic Polymer Composites (Terms Tech Publications, Zurich, 1994), Vol. 92-93, p. 217.

${ }^{8}$ H. Yamazaki and T. Kitayama, Ferroelectrics 33, 147 (1981).

${ }^{9}$ J. Yamada, T. Ueda, and T. Kitayama, J. Appl. Phys. 53, 4328 (1982).

${ }^{10}$ Y. G. Wang, W. L. Zhong, and P. L. Zhang, J. Appl. Phys. 53, 521 (1993).

${ }^{11}$ C. W. Nan, Phys. Rev. B 49, 12619 (1994).

${ }^{12}$ V. M. Levin and A. G. Luchaninov, J. Phys. D 34, 3058 (2001).

${ }^{13}$ J. Nye, Physical Properties of Crystals (Oxford University Press, London, U.K., 1979).

${ }^{14}$ R. M. Christensen, Mechanics of Composite Materials (Wiley, New York, 1979), Chap. 4.

${ }^{15}$ L. D. Landau and E. M. Lifshitz, Theory of Elasticity (ButterworthHeinemann, Washington, DC, 1997), Chap. 1.

${ }^{16}$ Z. Hashin, J. Appl. Mech. 29, 143 (1962).

${ }^{17}$ V. M. Levin, Mekh. Tela. 88, (1968) (in Russian).

${ }^{18}$ R. Coelho, Physics of Dielectrics for the Engineer (Elsevier Scientific, Amsterdam, 1979).

${ }^{19}$ B. Ploss, B. Ploss, F. G. Shin, H. L. W. Chan, and C. L. Choy, Proc. IEEE 1, 301 (2001).

${ }^{20}$ B. Ploss, B. Ploss, F. G. Shin, H. L. W. Chan, and C. L. Choy, Appl. Phys. Lett. 76, 2776 (2000).

${ }^{21}$ A. G. Bruggeman, Ann. Phys. (Leipzig) 24, 636 (1935).

${ }^{22}$ C. K. Wong, Y. M. Poon, and F. G. Shin, J. Appl. Phys. 90, 4690 (2001).

${ }^{23}$ A. S. Bhalla, R. E. Newnham, L. E. Cross, and W. A. Schulze, Ferroelectrics 33, 139 (1981).

${ }^{24}$ W. Y. Ng, Bernd Ploss, H. L. W. Chan, F. G. Shin, and C. L. Choy, Proc. IEEE 2, 767 (2001).

${ }^{25}$ G. Shirane and S. Hashino, J. Phys. Soc. Jpn. 6, 265 (1951). 